\title{
Artistic Analysis of Video Game Design Based on VR/AR Technology
}

\section{Zhu Lan}

Shougang Engineering College, Beijing, China, 100144

Keywords: VR; AR; video games; art taxonomy; new art

\begin{abstract}
This paper discusses the origin and purpose of art from the perspective of Virtual Reality and Augmented Reality technology. VR and AR games are introduced into the realm of art, and VR and AR are promoted to arts based on the innovative nature of the broad outline of the impact on the art world. Aiming at the above impact, this paper proposes a new classification method that is more suitable for the future development of the arts, that is, the logic classification to deal with the change that will be triggered after VR and AR games become an art form, briefly describes the significance of logical taxonomy for the development and creation of new art, illustrates the problems facing modern art through the Art Nouveau Manifesto and provides some additional explanations of previous issues.
\end{abstract}

\section{Introduction}

Virtual Reality is a comprehensive technology that integrates various technologies such as computer technology, multimedia technology, network technology and simulation technology [1]. Augmented Reality is a technique for calculating the position and angle of a camera image in real time and adding corresponding images. The goal of this technique is to set up and interact with the virtual world in the real world on the screen. The following Fig. 1 shows the Attributes of VR and AR.
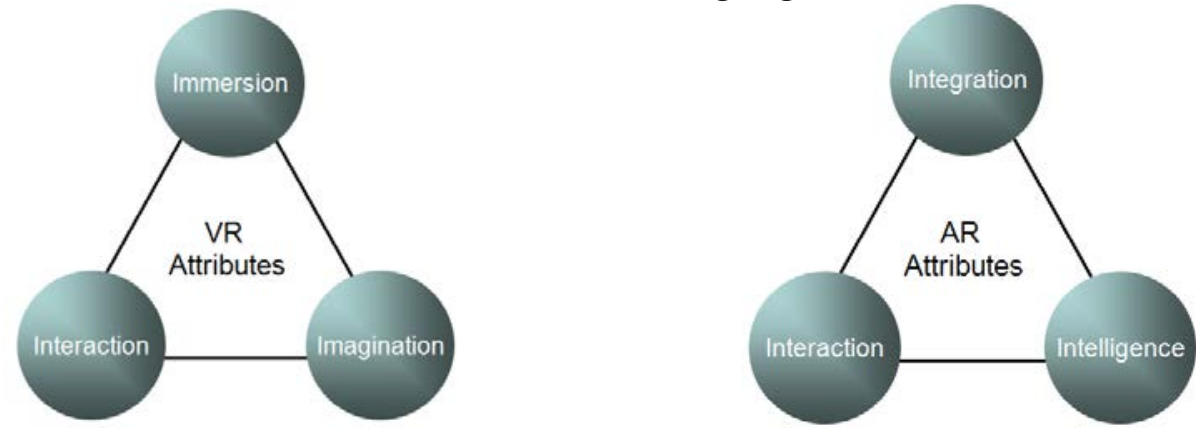

Fig. 1 Attributes of VR and AR

Video games, as a form of entertainment, have extraordinary charisma with their four major attributes. The VR, AR technology will undoubtedly further enhance the interactive and immersive video games. This is a completely new revolution for video games.

\section{VR/AR Games}

\subsection{Status quo VR/AR game}

VR/AR gaming wireless transmission technology is not yet mature, which leads to the game player a sense of vertigo, as well as the reaction delay in the game and other issues [2]. Action space is limited, which greatly restrict the freedom of the game. Full degree angle shooting is expensive and difficult, and full degree view image best known as pseudo VR, unable to achieve visual and auditory, olfactory, tactile sensory linkage are VR, AR games' flaw, which easily lead the game player out of the game and breaking its sense of immersion. Fig. 2 shows the comparison of five sources of humans sensory, which proved that view image, gives significant influent on players experience on VR/AR 
games.

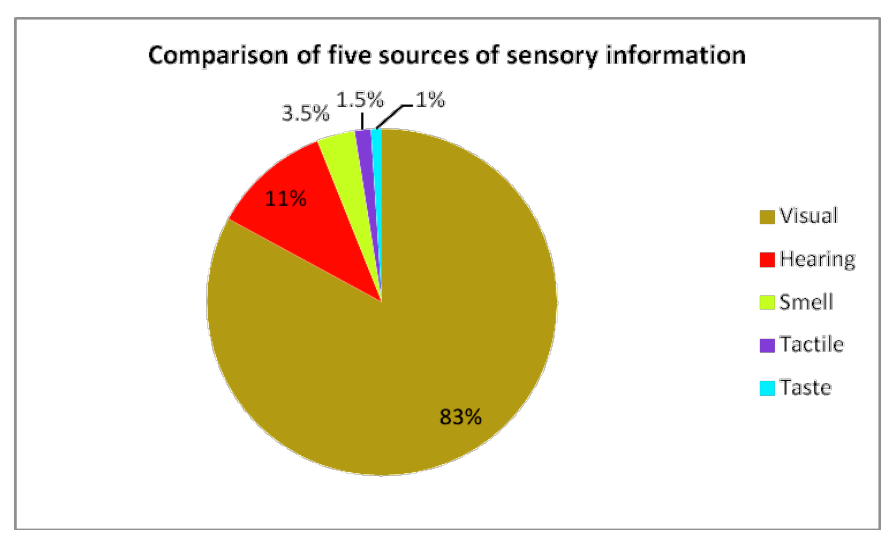

Fig. 2 Attributes of VR and AR

As a technology that will be popular to the general public, VR, AR products are too expensive and clumsy. VR, AR gaming hardware demanding, and now the public configuration is simply not enough to achieve a smooth and clear game experience. Moreover, without the support of mature communication technology, VR, AR games almost impossible to operate wirelessly, and can only wear dozens of data lines.

\subsection{Technology trends of VR/AR game}

The main reason for the year-on-year growth of the output value of the game industry is that the pace of modern life is getting faster and faster. More and more gamers aim to relax in the game, hoping to release their real-life pressure in the virtual world and even get rid of real life Have a sense of accomplishment. The traditional game visual experience effects are limited by the flat screen; the experience effect is not particularly ideal. VR, AR's technical characteristics is to enhance the visual perception and interactive games, regardless of their immersive experience or scene, the VR and AR combined games are far superior to traditional games.

But for the newly developed VR and AR game projects, it is far from enough to transplant only classic games. At present, the newly developed VR and AR game technology is not mature enough. With the rapid development of science and technology, the demand for virtual technology will be more and more severe. In the high cost and low performance VR and AR market, there is still a long way to go to meet the application needs of different game categories to VR and AR technology. At the same time, from the economic perspective, VR and AR hardware must reduce the cost of the economy and improve the performance of the game, which is the right way to develop the future. This requires the design of the game project to enhance the dynamic environment modeling technology, real-time three-dimensional graphics generation and display, augmented reality of the space environment, human-computer interaction more humane.

\section{The Artistry of VR/AR Games}

\subsection{VR/AR games belong to art.}

To determine whether a VR/AR game is a form of art or not, the key is whether it can achieve the purpose of Art - the transfer of emotions. In VR/AR games, players immerse themselves in the game and get rid of the merciless God's perspective. The feeling of participation makes the emotions more complex, real and intense. So VR/AR games belong to art. Table 1 shows the features of art and VR/AR games.

The seven major arts in the world are literature, music, dance, drama, painting, architecture, and film. This taxonomy is a customary classification of art. This sort of classification may be convenient in the past, but after the introduction of the VR and AR games to art, this classification method could be misleading. Since most of the past works of art can be re-expressed in VR/AR games. This expression, is not only the new wine in old bottles, but worthy of the name of creation. Plus, some 
new ways of expression, VR/AR games are quite a big part of the art.

Table 1 Comparison of Art and VR/AR games

\begin{tabular}{|c|c|c|}
\hline Items & Art & VR/AR Games \\
\hline Goals & Transfer emotions & Transfer emotions \\
\hline Interaction & Unidirectional & Bidirectional \\
\hline Feature & Need carrier & Re-expressed art \\
\hline
\end{tabular}

\subsection{Logical taxonomy of VR/AR games.}

An important feature of the past forms of art is expressing without interaction. In other words, it is the unilateral creation of the artistic creators, which also known as unidirectional art or expression art. With the assistant of VR and AR, electronic games become an art different from all previous mainstream art forms a two way interaction art. It is also called bi-directional art or interactive art [2]. In the unidirectional art, according to the difference of expression, it can be divided into direct Art and indirect Art. In the two way art, according to the difference of its purpose, it can be divided into Orienteering art and free art.

One way art in addition to creation also has appreciation, is not it interactive? That's not the case. In design art, we can regard its original as a table. The information contained in this table is finite and definite, and it is transmitted to the viewer unilaterally. And the viewer uses its own association and imagination to give the table some other meaning. This is the process of appreciation and thus produces specific emotions. However, the whole process of appreciation takes place on the one side of the viewer and is not transferred to the original, and thus cannot change the abstract emotions contained in the original. In simple terms, the viewer can't affect the original. So the art of design is a complete one-way art.

Abstract emotion is actually the emotion the author or performer wishes to express, and thus the emotion contained in the original or performance. The specific emotion refers to the emotion actually perceived by the viewer or the player. One way artwork infects viewers through abstract emotions, but the specific feelings generated by the viewer at the end can feel the influence of many factors. At the same time, abstract emotions cannot be clearly and precisely expressed because anyone's expression of it can only be an expression of the specific emotion that he has under his infection [3]. It's like when people look at something, they can only say what it looks like, but cannot say what it is.

The viewer, as an appreciator in the one-way art, emphasizes that he does not participate in the art creation and does not affect the original work. Players are the common elements of bi-directional art. As a participant in the two-way art, the player emphasizes his participation in the art creation and influences the original work.

\subsection{VR/AR games should not only focus on technology and ignore the artistry.}

VR/AR technology has brought people unprecedented visual experience, people's imagination to achieve a deeper level of development, the machine also has feelings, and humans can be more effective in the exchange and communication. In the meantime, the continuous advancement of digital technologies has reinvigorated the definition of games such as visual and audio experiences, and the novelty experience outperforms all kinds of games in the past.

The development of visual culture stems from the improvement of people's visual needs. As people are often imprisoned in image culture, people rely more and more on images, showing a gradual loss of artistic creativity and internal imagination. Digital art brings people a free channel of creation. The artistic features reflected in artistic language will highlight the characteristics of art, comprehensiveness, integration and authentic experience. Virtual art does not follow the imitation of the real environment in the initial stage of development, But to redefine the world, bringing people a 
number of perspectives, intuitive and real experience. Virtual art has changed the way of dealing with artistic conceptions in traditional art. The virtual artistic conception designs two-dimensional and three-dimensional multi-dimensional space through imagination, integrates the world into people's mind, re-establishes the space-time, the world and the environment, Imagination of the time and space reality.

\section{Summary}

Art will continue to infiltrate the factors of VR/AR science, science will continue to infiltrate the factors of art; eventually science and art will blend with each other or be independent of each other. The information technology is gradually reflected in all fields of society. VR/AR game design not only reflects the characteristics of spatial structure, but also aggravates the role of information carrier in terms of connotation, making it a communication platform that can connect public space with players. The design of VRAR games is a form of expression penetrating the digital media art in game design and also a cross-border cooperation product, demonstrating the future technological development for people. Therefore, the promotion of VR/AR technology can reflect the diversity of entertainment modes and provide people with a richer experience. VR/AR technology penetration into art products will bring a more perfect experience for people's artistic needs.

\section{References}

[1] Paul, Christiane, and Malcolm Levy. Genealogies of the New Aesthetic. Postdigital Aesthetics: Art, Computation and Design. Basingstoke: Palgrave Macmillan (2015), p.43

[2] Meixia Wang, Analysis of the Impact of VR and AR Technology on Digital Media Art, Art Technology 2016 (2), p.66

[3] Chen Ya Qian, Lei Kaibin. Virtual Reality Technology and Application [M]. Beijing: Science Press 2016, p. 25 